\title{
A short review on porous media convection
}

Ke Shao

School of Naval Architecture, Ocean 83 Civil Engineering, Shanghai Jiao Tong University, Shanghai 200240, China

\begin{abstract}
We briefly review the investigations of pattern formation and transport properties of RayleighDarcy convection (or the Elder Problem), including laboratory experiments, theoretical analysis and numerical simulations. It is shown that the flow exhibits power-law-scaling characteristics at large Rayleigh-Darcy number $R a$, a dimensionless parameter representing the ratio of the driving buoyancy forces to the diffusive forces. Namely, the mean spacing between neighboring interior plumes shrinks as $R a^{-\alpha}$ with the scaling exponent $\alpha \leq 1 / 2$ and the convective flux increases linearly with $R a$. However, more laboratory experiments are needed to validate these scalings. Additionally, many conditions, e.g. the inclination of the layer and hydrodynamic dispersion, etc., may lead to a large uncertainty in the flow pattern and transport efficiency.
\end{abstract}

Keywords: Porous media convection

\section{Introduction}

Buoyancy-driven convection in a fluid-saturated porous layer has been extensively studied due to its numerous applications in oil recovery [1], groundwater flow and geothermal energy extraction [2-7], transport in biological tissues [8], and carbon dioxide sequestration [9-23]. Moreover, porous media convection is also used as a classical example to study instabilities, bifurcations, pattern formation, and spatiotemporally chaotic dynamics [24-41].

In an infinitely-extended horizontal porous layer uniformly heated from the below and cooled from above, the system undergoes a series of bifurcations as the control parameter, the Rayleigh number $R a$ which represents the ratio of the driving buoyancy forces to the diffusive forces, increases. Above the critical Rayleigh number $R a=4 \pi^{2}$, the basic conduction state becomes linearly unstable via a stationary bifurcation and the flow exhibits steady convective rolls $[24,25]$. In a two-dimensional (2D) domain, the nonlinear steady rolls becomes unstable via a supercritical Hopf bifurcation at $R a \approx 400$, and small-scale protoplumes, generated from thin upper and lower boundary layers near the walls, are periodically advected around the $O(1)$ aspect-ratio cells $[28,31-33]$. For $400 \lesssim R a \lesssim 1300$, the flow transitions between periodic and quasiperiodic convective roll motions [28-33]; and for $R a \gtrsim 1300$, the large-scale convective rolls are completely broken down and the flow transitions to spatiotemporally chaotic motions [34, 36, 39]. 
At sufficiently large Rayleigh number, porous media convection exhibits a three-region columnar structure: adjacent to the upper and lower walls locate thin diffusive boundary layers; away from the walls the transport is controlled by nearly vertical columnar megaplumes; and between these two regions a series of protoplumes grow from the boundary layers and merge into the megaplumes $[34,36,39]$. As $R a$ is increased, the time-average inter-plume spacing $\delta$ decreases as a power of $R a, \delta \sim R a^{-\alpha}$, and the dimensionless transport flux $F$ (i.e. the ratio of the transport in the presence of convective motion to the diffusive transport, which is also referred to as the Nusselt number or the Sherwood number in many studies) increases as a power law of the form $F \sim R a^{\beta}$, where both $\alpha$ and $\beta$ are positive.

One of the central challenges in studies of porous media convection is the determination of the scaling exponents $\alpha$ and $\beta$. In this manuscript, we summarize various investigations from laboratory experiments, theoretical analysis and numerical simulations for studying the pattern formation and transport properties of porous media convection. At the end, we discuss the effects of various conditions on the convective pattern and transport.

\section{Experiments}

The laboratory experiments by Elder [42] in both bead packs and a Hele-Shaw cell first confirm that convection sets in when $R a \approx 4 \pi^{2}$, consistent with the theoretical prediction of $[24,25]$. The experiments by Lister [43] show that the lateral scale of the convective flow decreases with increasing Rayleigh number.

To explore the high- $R$ a scaling of convective transport in porous media, Neufeld et al. [14] perform laboratory experiments using solutions of methanol and ethylene-glycol mixing with water in a $2 \mathrm{D}$ chamber filled with glass beads. They find that the convective flux scales with the Rayleigh number to the $4 / 5$ power, i.e. $F \sim R a^{0.8}$, for $5 \times 10^{4} \leq R a \leq 6 \times 10^{5}$. Similarly, the experiments by Backhaus et al. [44] using solutions of water mixing with propylene glycol in a Hele-Shaw cell reveal that $F=0.045 R a^{0.76}$ for $6 \times 10^{3} \leq R a \leq 9 \times 10^{4}$.

\section{Theoretical analysis}

Linear stability analysis of the basic conduction solution indicates that the horizontal wavelength of the fastest growing disturbance decreases as $R a^{-1 / 4}$, while that of the smallest unstable mode scales as $R a^{-1 / 2}[24,25]$. Hence, to ensure the occurrence of convection, it requires $0<\alpha \leq 1 / 2$.

The 'marginally stable boundary layer' hypothesis by Malkus [45] yields a HowardMalkus-Kolmogorov-Spiegel scaling, $F \sim R a$, for porous media convection [46]. Moreover, rigorous upper-bound analyses of the Darcy-Oberbeck-Boussinesq equations by Doering and Constantin [46] and Otero et al. [34], by maximizing the heat transport of piecewise linear 'background' profiles, show that $F \leq c R a$ with different prefactors $c$. Following this strategy, [47-49] compute the optimal upper bound on $F$ under the Constantin-Doering-Hopf variational scheme. They find a lower prefactor $c$ for the unit optimal scaling exponent in the $F$-Ra relationship. Moreover, these upper-bound variational analysis also yields a set of a priori eigenfunctions which can be used to construct low-/reduced-order models for porous media convection [50-52]. 


\section{Numerical simulations}

The dynamics and flow pattern of porous media convection for low and moderate Rayleigh numbers have been thoroughly investigated theoretically and numerically, as described in the introduction. In this section, we focus on the dynamics for the 'turbulent' high- $R a$ regime.

At large values of Rayleigh number, convection in porous media appears in the form of three-region columnar flows, with a series of protoplumes growing from the thin upper and lower boundary layers and continuously merging with the interior megaplumes. The direct numerical simulations (DNS) of [36] show that the time-average inter-plume spacing in the 2D Rayleigh-Darcy system scales as $\delta \sim R a^{-0.4}$ and the interior flow can be modeled using a single horizontal Fourier-mode 'heat-exchanger' solution. Subsequently, they perform three-dimensional (3D) DNS in [38] and find that the high-Ra convection still retains the columnar structure in the 3D system, but with a different scaling for the time-average interplume spacing, i.e. $\alpha \approx 0.5$. To explore the mechanism for this nonlinear scale selection, $[36,38-40]$ perform linear stability analysis of the heat-exchanger solution and the nonlinear steady solution. They find that the mean inter-plume spacing $\delta$ observed at large $R a$ results from an interplay between two types of instability: when $\delta$ is too small, a bulk mode controls the instability, causing plume merger and coarsening of the convective pattern; when $\delta$ is too large, a wall-mode instability dominates, causing small plumes generated from the walls to split the wider plumes into narrower ones. Moreover, all of these studies reveal that as $R a \rightarrow \infty$, the convective flux increases linearly with $R a$ for both $2 \mathrm{D}$ and $3 \mathrm{D}$ systems, i.e. $\beta=1$.

\section{Conditions affecting the pattern and transport}

In previous sections, we summarize various studies of the convective pattern and transport properties in the Rayleigh-Darcy domain. Most of these studies are performed in simplified/idealized geometries. In this section, we discuss several conditions which may affect the flow pattern and transport efficiency of porous media convection.

\subsection{Domain aspect ratio}

The small domain aspect ratio $L$, i.e. the ratio of the horizontal to the vertical dimension of the container, may significantly affect the convective pattern and transport. When $L$ is smaller than the smallest unstable wavelength $4 \pi /\left(\sqrt{R a}+\sqrt{R a-4 \pi^{2}}\right)$ in the horizontal Rayleigh-Darcy domain, no convection occurs due to the stable conduction state [24, 25]. In the thermal convection problem, to obtain a reliable statistical results for heat transport flux, the domain should contain at least one sustained megaplume [48, 51].

\section{2. $2 D$ v.s. $3 D$}

For simplification, most of the current studies on porous media convection are performed in the $2 \mathrm{D}$ systems. Although an extra freedom in 3D may lead to more complicated convective patterns and affect the transport efficiency, the studies by $[38,53,54]$ show that 
in $3 \mathrm{D}$ the flow still retains a similar columnar structure at large $R a$ in horizontal porous layers, and the convective flux increases linearly with $R a$ but with a slightly larger prefactor. Therefore, the analysis in 2D does, indeed, shed light on the real 3D system.

\subsection{Inclination of layer}

In a sloping 3D porous layer, the studies by [55-61] indicate that the conduction state is stable for $R a \cos \phi \leq 4 \pi^{2}$, where $\phi$ is the inclination angle above the horizontal, and four types of flow exist near the onset of convection for different $\phi$ : the basic unicellular flow (i.e. the conduction state); polyhedral cells; longitudinal helicoidal cells; and 2D transverse rolls. The experimental studies by $[62,63]$ reveal that a small amount of slope of layer enhances the convective transport compared to the horizontal case.

In the inclined 2D porous layer, the non-convective basic state at large $R a$ is linearly stable for $\phi \geq 31.3^{\circ}$ [64]. However, it is shown in [65] that the basic state is not energy stable so that convective states may be realizable. In fact, the DNS results in $[65,66]$ indicate that for $0<\phi<25^{\circ}$, the flow still exhibits the three-region wall-normal columnar structure as in the horizontal case; while for $\phi>32^{\circ}$, the flow transitions to a large-scale traveling-wave convective roll state, and the heat transport is significantly reduced.

\subsection{Hydrodynamic dispersion}

As large Rayleigh number, the strong convection leads to fast small-scale roll motions. When the flow velocity is large enough, the mechanical dispersion may dominate the convection [67-69]. Recent studies by [70-80] indicate that at large $R a$ hydrodynamic dispersion significantly affects the flow pattern and transport of convection in porous media. Although most of the studies agree that hydrodynamic dispersion coarsens the convective pattern, $[71,72]$ show that the hydrodynamic dispersion enhances the convective mixing, while the laboratory experiments by $[75,77]$ reveal that it reduces the increase of convective flux with increasing permeability.

\subsection{Anisotropy}

Due to the inherent anisotropy of many sedimentary geological formations, it is necessary to explore the effect of anisotropic permeability on convection. [10, 11, 81, 82] investigate the onset of convection in anisotropic porous media with a vertical-to-horizontal permeability ratio $\gamma=k_{v} / k_{h}$, and find that the anisotropic system $(\gamma<1)$ becomes more unstable compared with the isotropic one. The DNS results from [83] for $0.25 \leq \gamma \leq 1$ up to $R a=4 \times 10^{4}$ show that the anisotropic permeability does not change the unit exponent in the $F$ - $R a$ scaling relationship at large $R a$ but increases the prefactor.

\section{Summary}

We have briefly reviewed various investigations of flow pattern and transport characteristics of convection in porous media, including laboratory experiments, theoretical analysis and numerical simulations. The theoretical analysis and numerical simulations show that in a sufficiently extended porous layer and as $R a \rightarrow \infty$, the inter-plume spacing shrinks 
as a power-law scaling of $R a$ with the scaling exponent $\alpha \leq 1 / 2$ and the scaling exponent in the $F-R a$ relationship is unity. However, these scalings have not been verified due to the lack of laboratory data. Moreover, at large Rayleigh number many conditions, e.g. the inclination of the layer and hydrodynamic dispersion, etc., may lead to a large uncertainty in the pattern formation and transport efficiency.

\section{References}

[1] T. K. Perkins, O. C. Johnston, A review of diffusion and dispersion in porous media, Society of Petroleum Engineers (1963).

[2] O. M. Phillips, Flow and Reactions in Permeable Rocks, Cambridge University Press, 1991.

[3] O. M. Phillips, Geological Fluid Dynamics: Sub-surface Flow and Reactions, Cambridge University Press, 2009.

[4] I. Pop, D. B. Ingham, Convective heat transfer: mathematical and computational modeling of viscous fluids and porous media, Elsevier, 2001.

[5] D. A. Nield, A. Bejan, Convection in Porous Media, Springer, New York, 3rd edn edition, 2006.

[6] Y. Liang, J. Sheng, J. Hildebrand, Dynamic permeability models in dual-porosity system for unconventional reservoirs: Case studies and sensitivity analysis, in: SPE Reservoir Characterisation and Simulation Conference and Exhibition, Society of Petroleum Engineers, Abu Dhabi, UAE, May, 2017.

[7] Y. Liang, B. Yuan, A guidebook of carbonate laws in china and kazakhstan: Review, comparison and case studies, Carbon Management Technology Conference, Houston, USA, July, 2017.

[8] A.-R. Khaled, K. Vafai, The role of porous media in modeling flow and heat transfer in biological tissues, Int. J. Heat Mass Transfer 46 (2003) 4989-5003.

[9] B. Metz, O. Davidson, H. de Coninck, M. Loos, L. Meyer, IPCC Special Report on Carbon Dioxide Capture and Storage, Cambridge University Press, New York, 2005.

[10] J. Ennis-King, I. Preston, L. Paterson, Onset of convection in anisotropic porous media subject to a rapid change in boundary conditions., Phys. Fluids 17 (2005) 084107.

[11] X. Xu, S. Chen, D. Zhang, Convective stability analysis of the long-term storage of carbon dioxide in deep saline aquifers., Adv. Water Resour. 29 (2006) 397-407.

[12] A. Riaz, M. Hesse, H. A. Tchelepi, F. M. O. Jr, Onset of convection in a gravitationally unstable diffusive boundary layer in porous media., J. Fluid Mech. 548 (2006) 87-111.

[13] M. Kim, K. Song, C. Choi, J.-K. Yeo, Onset of buoyancy-driven convection in a liquid-saturated cylindrical porous layer supported by a gas layer, Phys. Fluids 20 (2008) 054104.

[14] J. A. Neufeld, M. A. Hesse, A. Riaz, M. A. H. andH. A. Tchelepi, H. E. Huppert, Convective dissolution of carbon dioxide in saline aquifers., Geophys. Res. Lett. 37 (2010) L22404.

[15] J. J. Hidalgo, J. Fe, L. Cueto-Felgueroso, R. Juanes, Scaling of convective mixing in porous media., Phys. Rev. Lett. 109 (2012) 264503.

[16] M. Szulczewski, M. Hesse, R. Juanes, Carbon dioxide dissolution in structural and stratigraphic traps, J. Fluid Mech. 736 (2013) 287-315.

[17] D. R. Hewitt, J. A. Neufeld, J. R. Lister, Convective shutdown in a porous medium at high rayleigh number., J. Fluid Mech. 719 (2013) 551-586.

[18] A. C. Slim, Solutal-convection regimes in a two-dimensional porous medium., J. Fluid Mech. 741 (2014) 461-491.

[19] K. J. Sathaye, M. A. Hesse, M. Cassidy, D. F. Stockli, Constraints on the magnitude and rate of $\mathrm{CO}_{2}$ dissolution at Bravo Dome natural gas field., Proc Natl Acad Sci (PNAS). 111 (2014) 15332-15337.

[20] N. Tilton, A. Riaz, Nonlinear stability of gravitationally unstable, transient, diffusive boundary layers in porous media, J. Fluid Mech. 745 (2014) 251-278.

[21] B. Wen, D. Akhbari, L. Zhang, M. A. Hesse, Convective carbon dioxide dissolution in a closed porous medium at low pressure, J. Fluid Mech. 854 (2018) 56-87.

[22] D. Akhbari, M. A. Hesse, Causes of underpressure in natural $\mathrm{CO}_{2}$ reservoirs and implications for geological storage, Geology 45 (2017) 47-50. 
[23] Z. Shi, B. Wen, M. Hesse, T. Tsotsis, K. Jessen, Measurement and modeling of $\mathrm{CO}_{2}$ mass transfer in brine at reservoir conditions, Advances in Water Resources 113 (2018) 100-111.

[24] C. W. Horton, F. T. Rogers, Convection currents in a porous medium, J. Appl. Phys. 16 (1945) 367-370.

[25] E. R. Lapwood, Convection of a fluid in a porous medium, Proc. Camb. Phil. Soc. 44 (1948) 508-521.

[26] R. A. Wooding, Steady state free thermal convection of liquid in a saturated permeable medium, Journal of Fluid Mechanics 2 (1957) 273285.

[27] R. A. Wooding, Convection in a saturated porous medium at large rayleigh number or peclet number, Journal of Fluid Mechanics 15 (1963) 527544.

[28] G. Schubert, J. M. Straus, Transitions in time-dependent thermal convection in fluid-saturated porous media, J. Fluid Mech. 121 (1982) 301-313.

[29] S. Kimura, G. Schubert, J. M. Straus, Route to chaos in porous-medium thermal convection, J. Fluid Mech. 166 (1986) 305-324.

[30] S. Kimura, G. Schubert, J. M. Straus, Instabilities of steady, periodic, and quasi-periodic modes of convection in porous media, J. Heat Transfer 109 (1987) 350-355.

[31] C. K. Aidun, P. H. Steen, Transition to oscillatory convective heat transfer in a fluid-saturated porous medium, J. Thermophys. H. T. 1 (1987) 268-273.

[32] M. D. Graham, P. H. Steen, Strongly interacting traveling waves and quasiperiodic dynamics in porous medium convection, Physica D 54 (1992) 331-350.

[33] M. D. Graham, P. H. Steen, Plume formation and resonant bifurcations in porous-media convection, J. Fluid Mech. 272 (1994) 67-90.

[34] J. Otero, L. A. Dontcheva, H. Johnston, R. A. Worthing, A. Kurganov, G. Petrova, C. R. Doering, High-Rayleigh-number convection in a fluid-saturated porous layer., J. Fluid Mech. 500 (2004) 263-281.

[35] D. Rees, A. Barletta, Linear instability of the isoflux Darcy-Bénard problem in an inclined porous layer, Transp. Porous Media 87 (2011) 665-678.

[36] D. R. Hewitt, J. A. Neufeld, J. R. Lister, Ultimate regime of high Rayleigh number convection in a porous medium., Phys. Rev. Lett. 108 (2012) 224503.

[37] D. R. Hewitt, J. A. Neufeld, J. R. Lister, Stability of columnar convection in a porous medium, J. Fluid Mech. 737 (2013) 205-231.

[38] D. R. Hewitt, J. A. Neufeld, J. R. Lister, High rayleigh number convection in a three-dimensional porous medium, J. Fluid Mech. 748 (2014) 879-895.

[39] B. Wen, L. T. Corson, G. P. Chini, Structure and stability of steady porous medium convection at large Rayleigh number, J. Fluid Mech. 772 (2015) 197-224.

[40] D. R. Hewitt, J. R. Lister, Stability of three-dimensional columnar convection in a porous medium, J. Fluid Mech. 829 (2017) 89-111.

[41] B. Wen, M. Hesse, Rayleigh fractionation in high-Rayleigh-number solutal convection in porous media, arXiv preprint arXiv:1801.03075 (2018).

[42] J. W. Elder, Steady free convection in a porous medium heated from below, Journal of Fluid Mechanics 27 (1967) 2948.

[43] C. R. B. Lister, An explanation for the multivalued heat transport found experimentally for convection in a porous medium, Journal of Fluid Mechanics 214 (1990) 287320.

[44] S. Backhaus, K. Turitsyn, R. E. Ecke, Convective instability and mass transport of diffusion layers in a hele-shaw geometry, Phys. Rev. Lett. 106 (2011) 104501.

[45] W. V. R. Malkus, The heat transport and spectrum of thermal turbulence, Proc. R. Soc. Lond. A225 (1954) 196-212.

[46] C. R. Doering, P. Constantin, Bounds for heat transport in a porous layer, J. Fluid Mech. 376 (1998) 263-296.

[47] B. Wen, N. Dianati, E. Lunasin, G. P. Chini, C. R. Doering, New upper bounds and reduced dynamical modeling for Rayleigh-Bénard convection in a fluid saturated porous layer, Communications in Nonlinear Science and Numerical Simulation 17 (2012) 2191-2199.

[48] B. Wen, G. P. Chini, N. Dianati, C. R. Doering, Computational approaches to aspect-ratio-dependent 
upper bounds and heat flux in porous medium convection, Phys. Lett. A 377 (2013) 2931-2938.

[49] B. Wen, G. P. Chini, R. R. Kerswell, C. R. Doering, Time-stepping approach for solving upper-bound problems: Application to two-dimensional Rayleigh-Bénard convection, Phys. Rev. E 92 (2015) 043012.

[50] G. P. Chini, N. Dianati, Z. Zhang, C. R. Doering, Low dimensional models from upper bound theory, Physica D 240 (2011) 241-248.

[51] N. Dianati, Reduced-Dimensional Models of Porous-Medium Convection, Ph.D. thesis, University of Michigan, 2013.

[52] B. Wen, G. P. Chini, Reduced modeling of porous media convection in a minimal flow unit at large Rayleigh number, Journal of Computational Physics 371 (2018) 551-563.

[53] G. S. Pau, J. B. Bell, K. Pruess, A. S. Almgren, M. J. Lijewski, K. Zhang, High-resolution simulation and characterization of density-driven flow in $\mathrm{CO}_{2}$ storage in saline aquifers., Adv. Water Resour. 33 (2010) 443455.

[54] X. Fu, L. Cueto-Felgueroso, R. Juanes, Pattern formation and coarsening dynamics in three-dimensional convective mixing in porous media, Phil Trans R Soc A 371 (2013) 20120355.

[55] J. P. Caltagirone, M. Cloupeau, M. Combarnous, Convection naturelle fluctuante dans une couche poreuse horizontale, C. R. Acad. Sci. Paris B 273 (1971) 833-836.

[56] S. A. Bories, M. A. Combarnous, J. Jaffrenou, Observations des différentes formes d'écoulements thermoconvectifs dans une couche poreuse inclinée, C. R. Acad. Sci. Paris A 275 (1972) 857-860.

[57] S. A. Bories, L. Monferran, Condition de stabilité et échange thermique par convection naturelle dans une couche poreuse inclinée de grande extension, C. R. Acad. Sci. Paris B 274 (1972) 4-7.

[58] S. A. Bories, M. A. Combarnous, Natural convection in a sloping porous layer, J. Fluid Mech. 57 (1973) 63-79.

[59] T. Kaneko, An Experimental Investigation of Natural Convection in Porous Media, M.Sc. Thesis, University of Calgary, 1972.

[60] T. Kaneko, M. F. Mohtadi, K. Aziz, An experimental study of natural convection in inclined porous media, Int. J. Heat Mass Transfer 17 (1974) 485-496.

[61] J. P. Caltagirone, S. Bories, Solutions and stability criteria of natural convective flow in an inclined porous layer, J. Fluid Mech. 155 (1985) 267-287.

[62] C. W. MacMinn, R. Juanes, Buoyant currents arrested by convective dissolution, Geophysical Research Letters 40 (2013) 2017-2022.

[63] P. A. Tsai, K. Riesing, H. A. Stone, Density-driven convection enhanced by an inclined boundary: Implications for geological $\mathrm{CO}_{2}$ storage, Physical Review E 87 (2013) 011003.

[64] D. Rees, A. Bassom, The onset of Darcy-Bénard convection in an inclined layer heated from below, Acta Mech. 144 (2000) 103-118.

[65] B. Wen, G. P. Chini, Inclined porous medium convection at large Rayleigh number, J. Fluid Mech. 837 (2018) 670-702.

[66] B. Wen, Porous medium convection at large Rayleigh number: Studies of coherent structure, transport, and reduced dynamics, Ph.D. thesis, University of New Hampshire, Durham, 2015.

[67] J. Bear, On the tensor form of dispersion in porous media., J. Geophys. Res. 66 (1961) 1185-1197.

[68] G. de Josselin de Jong, M. J. Bossen, Discussion of paper by Jacob Bear, "On the tensor form of dispersion in porous media"., J. Geophys. Res. 66 (1961) 3623-3624.

[69] A. E. Scheidegger, General theory of dispersion in porous media., J. Geophys. Res. 66 (1961) 3273-3278.

[70] K. Ghesmat, J. Azaiez, Viscous fingering instability in porous media: Effect of anisotropic velocitydependent dispersion tensor., Transp. Porous Med. 73 (2008) 4297-318.

[71] J. J. Hidalgo, J. Carrera, Effect of dispersion on the onset of convection during $\mathrm{CO}_{2}$ sequestration, J. Fluid Mech. 640 (2009) 441-452.

[72] K. Ghesmat, H. Hassanzadeh, J. Abedi, The effect of anisotropic dispersion on the convective mixing in long-term $\mathrm{CO}_{2}$ storage in saline aquifers., AIChE J 57 (2011) 561-570.

[73] Y. Liang, Experimental study of convective dissolution of carbon dioxide in porous media, Master's thesis, The University of Texas at Austin, 2014.

[74] H. Emami-Meybodi, H. Hassanzadeh, J. Ennis-King, $\mathrm{CO}_{2}$ dissolution in the presence of background 
flow of deep saline aquifers., Water Resour. Res. 51 (2015) 2595-2615.

[75] L. Wang, Y. Nakanishi, A. Hyodo, T. Suekane, Three-dimensional structure of natural convection in a porous medium: Effect of dispersion on finger structure, Int. J. Greenh. Gas Control 53 (2016) 274-283.

[76] T. Suekane, J. Ono, A. Hyodo, Y. Nagatsu, Three-dimensional viscous fingering of miscible fluids in porous media, Phys. Rev. Fluids 2 (2017) 103902.

[77] Y. Liang, Scaling of Solutal Convection in Porous Media, Ph.D. thesis, The University of Texas at Austin, 2017.

[78] Y. Liang, Y. Ning, L. Liao, B. Yuan, Chapter fourteen - special focus on produced water in oil and gas fields: Origin, management, and reinjection practice, in: B. Yuan, D. A. Wood (Eds.), Formation Damage During Improved Oil Recovery, Gulf Professional Publishing, 2018, pp. 515 - 586.

[79] B. Wen, K. W. Chang, M. A. Hesse, Convection in porous media with dispersion, arXiv preprint arXiv:1803.01270 (2018).

[80] Y. Liang, B. Wen, M. Hesse, D. DiCarlo, Effect of dispersion on solutal convection in porous media, Geophys. Res. Lett. (2018).

[81] D. Rees, A. Postelnicu, The onset of convection in an inclined anisotropic porous layer, Int. J. Heat Mass Transfer 44 (2001) 4127-4138.

[82] P. Cheng, M. Bestehorn, A. Firoozabadi, Effect of permeability anisotropy on buoyancydriven flow for $\mathrm{CO}_{2}$ sequestration in saline aquifers, Water Resources Research 48 (2012) W09539.

[83] M. D. Paoli, F. Zonta, A. Soldati, Influence of anisotropic permeability on convection in porous media: Implications for geological $\mathrm{CO}_{2}$ sequestration, Physics of Fluids 28 (2016) 056601. 